\title{
Fiber Reinforced Composite Materials for Proton Radiation Shielding
}

\author{
MIHAELA RALUCA CONDRUZ1*, CRISTIAN PUSCASU ${ }^{1}$, LUCIA RALUCA VOICU ${ }^{1}$, IONUT SEBASTIAN VINTILA ${ }^{1}$, \\ ALEXANDRU PARASCHIV ${ }^{1}$, DRAGOS ALEXANDRU MIREA ${ }^{2}$ \\ ${ }^{1}$ COMOTI Institute of Reserch\&Development Turboengines, 220 D Iuliu Maniu Av., Bucharest, Romania \\ ${ }^{2}$ Horia Hulubei National Institute for R\&D in Physics and Nuclear Engineering, 30 Reactorului Str., Magurele, Romania
}

\begin{abstract}
Nowadays scientific researchers aim to develop new material designs for space structures thatcan withstand the harsh conditions in space environment. Another goal is to reduce the weight and the launching cost of the structures. A solution to those needs is to integrate fiber reinforced polymers in spacecraft structural components. Radiation shielding is a requirement that has to be met by the materials used to manufacture space structures. Protons are one of the many charged particles that can influence the integrity of materials in space. In the present study three material designs were developed and their ability to shield proton beam irradiation was evaluated. The material designs consist in advanced composite materials (carbon fiber reinforced polymers - CFRPs) that integrate the concept Low Z - High Z - Low Z (tantalum foil) and metallic coatings. Simulations were performed to determine the penetration depth of an ion beam (energy $15 \mathrm{MeV}$ ) in the proposed material designs. It was observed that the beam's penetration depth through a CFRP sample with Ta foil (sample's thickness $2.08 \mathrm{~mm}$ ) was about 75\% of the sample's thickness, $58 \%$ for CFRP sample with Babbitt coating (sample's thickness $2.28 \mathrm{~mm}$ ), 56\% for the CFRP sample with Zn coating (sample's thickness $2.28 \mathrm{~mm}$ ) and 55\% for the CFRP sample with Zn/Monel coating (sample's thickness $2.28 \mathrm{~mm}$ ). It seems that the proposed material designs provide ion beam protection similar with an aluminum sample of $2 \mathrm{~mm}$ thickness. The experimental procedure confirmed the results obtained from the simulations, all the material designs providing protection in case of proton beam irradiation with an energy of $15.8 \mathrm{MeV}$.
\end{abstract}

Keywords: advanced composites, carbon fiber reinforced polymer (CFRP), radiation shielding, proton irradiation

Nowadays scientific researchers aim to develop new materials with better properties and new structures for space exploration with specific designs and characteristics that can withstand the harsh space conditions. Radiation shielding is an important challenge for the material designers. The purpose of a shielding material is to attenuate the energy and the flux of ionizing radiation as they pass through the shield material. The ionizing radiation can be defined as any type of radiation consisting of charged particles or uncharged particles or both, that, as a result of physical interaction, creates ions of opposite signs by either primary or secondary processes [1]. The general radiation environment in space from all sources is referred to as cosmic radiation. Cosmic radiation is comprised of various charged particles, including protons, electrons, and ions of heavy elements with a range of energies and fluences depending on many factors such as spacecraft trajectory, time of the solar cycle, and rare solar events [2]. In space are three main sources of radiation: Trapped Radiation Belts (TRB) or Van Allen Radiation Belts, Sun Cosmic Rays (SCR) and Galactic Cosmic Rays (GCR). The inner Van Allen radiation belt comprises protons within the energy range of $0.1 \div 400 \mathrm{MeV}$ and electrons with energies lower then $0.78 \mathrm{MeV}$. The outer Van Allen radiation belt comprises electrons within the energy range of $0.04 \div 7$ MeV [4]. The Sun Cosmic Rays are caused by Solar Particle Events (SPE). A SPE occurs when particles emitted by the sun are accelerated by a solar flare or the shock wave associated with a coronal mass ejection (CME) [5]. CMEs are large eruptions of proton-rich plasma (a gas of free ions and electrons) that drive a shock wave outwards and accelerate particles to ultrasonic speeds of $50-1200 \mathrm{~km} \cdot \mathrm{s}^{\prime \prime}$ in the process [6]. The Coronal Mass ejection consists in $96.4 \%$ protons, $3.5 \%$ alpha particles and $0.1 \%$ heavy ions
[4]. The Galactic Cosmic Radiation (GCR) consists of charged particles originating from beyond the Solar system. They are of somewhat mysterious origin, but there is evidence that a significant portion of GCR comes from supernovae of massive stars [7]. The GCR composition is proportional to solar system material: $98 \%$ hadrons and 2 $\%$ leptons. The hadrons are subdivided in $87 \%$ protons, $12 \%$ al pha particles and $1 \%$ heavy ions [4]. Trapped protons are the most important source of radiation for LEO missions with an altitude around $450 \mathrm{~km} \mathrm{[3].} \mathrm{According} \mathrm{to} \mathrm{[1]} \mathrm{the}$ energy ranges for the protons on LEO and POL orbits are within $100 \mathrm{keV}$ to $200 \mathrm{MeV}$.

In the past, to increase the shielding capability of one structure the engineers were just adding more aluminium to the component. Aluminum is a good structural material, but it is relatively heavy, which is a major concern for space flights, as each extra kilogram increases the mission cost [8] (e.g. in its early operational stage, launching $1 \mathrm{~kg}$ on LEO with Vega launcher cost $\$ 23.300$ [9]).

A solution to reduce the structure's mass and launching costs was to integrate fiber reinforced polymers (FRP) in spacecraft structural components. Polymeric composite materials reinforced with fibers (high performance fibers like carbon or aramid fibers) have specific strength (strength per unit weight) and stiffness, corrosion and fatigue resistance, tailorable conductivity, controlled thermal expansion and the ability to be processed into complex shapes [10]. The concept of radiation shielding has been researched in the last decades. Mangeret et al. [11] showed that low-Z materials (materials with low atomic numbers Z) attenuate protons better than their high$Z$ counterparts. Electrons and photons were attenuated more effectively by high-Z materials. In case both protons and electrons were needed to be attenuated bilayer 
concepts were more effective (a high-Z layer on the outside and a low-Z layer on the inside). The optimum thickness of the high-Z layer depends on the radiation environment, such that for electron-rich orbits a larger quantity of high-Z materials is required.

Axtega et.al. [10] studied the radiation shielding obtained by the incorporation of nanomaterials (carbon nanotubes and tungsten nanoparticles) in composite structures. They performed simulation and experimental tests on proton, electron and gamma irradiation. The proton simulation were made in the region of $1-100 \mathrm{MeV}$ due to the fact that most of the effect of thin shielding materials will occur in the lower end of the selected energy region. The proton irradiation was realised at $20 \mathrm{MeV}$ protons beam under vacuum. They observed that the simulation followed closely the test results for proton irradiation $(20 \mathrm{MeV})$ and the tungsten (high Z material) has the highest effect on kinetic energy of the incoming protons. At lower energies, layers made from prepregs have equal effect on the particle energies as $50 \mu \mathrm{m}$ of tungsten.

Brander et.al. [12] made some radiation attenuation calculations and simulations on CFRP material designs integrating the concept Low Z - High Z - Low Z and they performed tests to verify the analyses results. The CFRP material designs consisted in epoxy matrix prepreg reinforced with carbon fiber. Total of four samples were manufactured, three of these had wolfram and one was pure CFRP laminate, one aluminum reference was added to the tests. They made proton and electron irradiation tests. The nominal irradiation levels for electrons were $1.5 \mathrm{MeV}$, $2 \mathrm{MeV}, 3 \mathrm{MeV}$ and $5 \mathrm{MeV}$ and for protons $15 \mathrm{MeV}, 17.5 \mathrm{MeV}$ and $20 \mathrm{MeV}$. The levels were derived from the radiation profile of PROBA satellite. The applied concept provided better or equivalent protection against the electrons as 2 $\mathrm{mm}$ of aluminum at studied energy ranges, however it seemed that even the double thickness of wolfram cannot provide as good protection against protons than $2 \mathrm{~mm}$ of aluminum.

\section{Experimental part}

\section{Sample manufacturing}

Three material design configurations were under study along with a reference, the material configurations can be observed in table 1. All the samples were manufactured from carbon reinforced polymer (CFRP) prepreg by the autoclave technology (the curing cycle along with the vacuum bag assembly are presented in fig. 1). The prepreg consisted in a 3K HTA carbon fiber fabric twill $2 \times 2$ preimpregnated with an epoxy cyanate ester blend (Gurit EP 127-C20-46P T2x2). The material designs comprised the concept Low Z - High Z - Low Z, the high Z material selected was a tantalum foil $(99.95 \% \mathrm{Ta}$ and $0.08 \mathrm{~mm}$ thickness) and a metallic coating ( $200 \mu \mathrm{m}$ of $\mathrm{Zn}, 200 \mu \mathrm{m}$ of Babbitt or a graded coating $100 \mu \mathrm{m} \mathrm{Zn/} \mathrm{100 \mu m} \mathrm{Monel).}$ To ensure physical bonding between the metallic foil and the prepreg plies, the tantalum foil was sandblasted with F33 corundum in two stages (first 1 min at a pressure of 3 bar and then 1 min at a pressure of 5 bar) and degreased with acetone. In figure 2 can be observed microscopic images with foil surface before and after the blasting.

After the curing, the laminates were removed from the moulds and then were sandblasted with F30 corundum ( 6 stages blasting, pressure: 4 bar, distance from the sample: $100 \mathrm{~mm}$ ). The polymeric substrates were coated using Zn, Babbitt and Zn/Monel by thermal spray technology (arc spray technique) and 15x15 mm samples were cut from the composite laminates with a water jet cutting machine (pressure 4000 bar).

\section{Proton irradiation simulation}

Proton irradiation simulations were made to determinate the penetration depth of a ion beam in the material designs. The simulations were made with SRIM programme (Stopping and Range of lons in Matter), a programme which calculates interactions of ions with matter. It is based on a Monte Carlo simulation method (binary collision approximation). Different types of configuration samples were used as input for the simulation, along with their
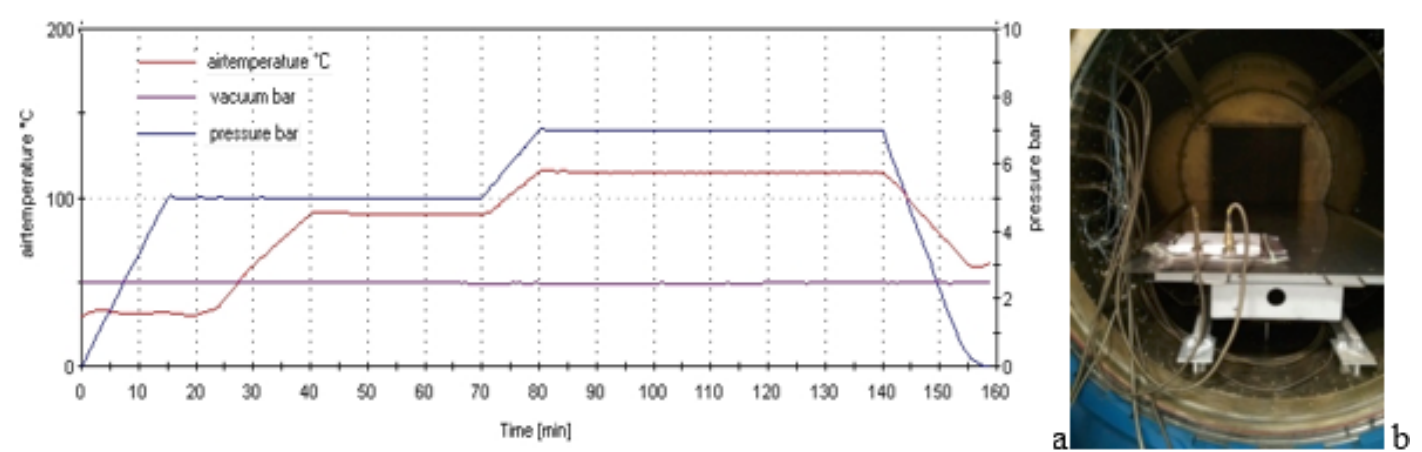

Fig. 1. a) The curing cycle of the polymeric composites; b) The vacuum bag assembly

\begin{tabular}{|l|l|c|}
\hline Sample code & \multicolumn{1}{|c|}{ Configuration } & Sample dimensions \\
\hline Reference & 8 plies CFRP & $2 \mathrm{~mm}$ \\
\hline Design 1 & 4 plies CFRP/ Ta foil/4 plies CFRP/Zn & $2.28 \mathrm{~mm}$ \\
\hline Design 2 & 4 plies CFRP/ Ta foil/4 plies CFRP/ Babbitt & $2.28 \mathrm{~mm}$ \\
\hline Design 3 & 4 plies CFRP/ Ta foil/ 4 plies CFRP/Zn/Monel & $2.28 \mathrm{~mm}$ \\
\hline
\end{tabular}
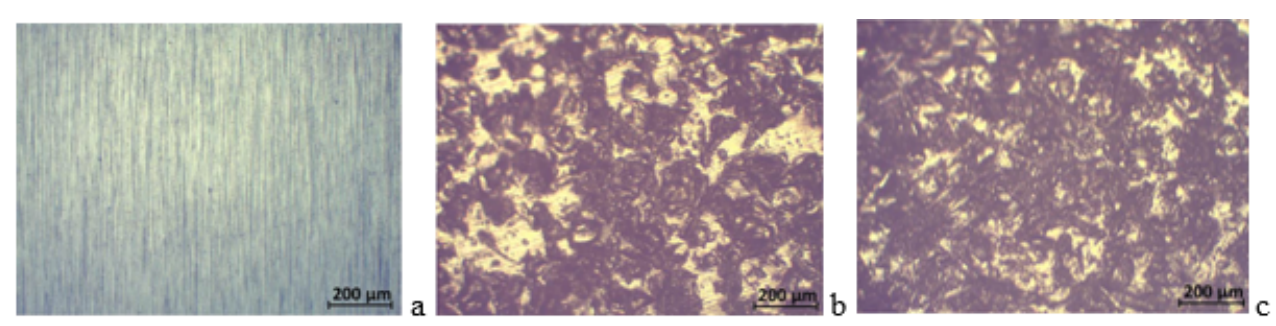

Fig. 2. Tantalum foil before and after the sandblasting: a) microscopic image with the foil before sandblasting (magnification 100x); b) microscopic image with the foil after the first sandblasting stage (magnification 100x); c) microscopic image with the foil after the second sandblasting stage (magnification 

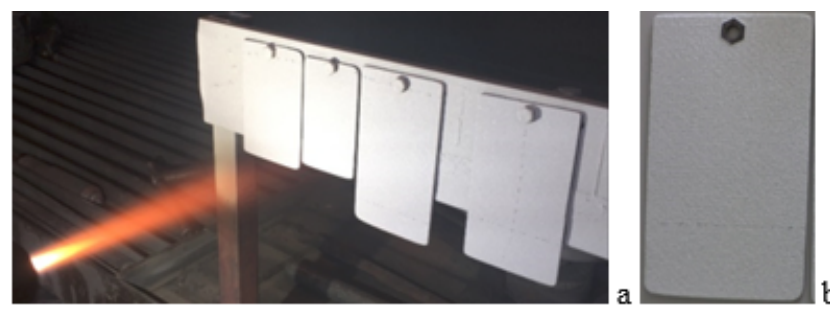

chemical composition, density, thickness, ion type and beam's energy.

The samples used as input data were:

- aluminum sample $2 \mathrm{~mm}$ thick;

- CFRP sample $2 \mathrm{~mm}$ thick;

- CFRP sample $1.9 \mathrm{~mm}$ thick;

- CF.08 mm thick Ta foil) $2.08 \mathrm{~mm}$ thick;

- CFRP sample comprising the Low Z - High Z -Low Z

concept and a $200 \mu \mathrm{m}$ thick $Z n$ coating $2.28 \mathrm{~mm}$ thick;

- CFRP sample comprising the Low Z -High Z- Low Z conceptand a $200 \mu \mathrm{m}$ thick Babbitt coating $2.28 \mathrm{~mm}$ thick; - CFRP sample integrating the Low Z - High Z - Low Z concept and a $100 \mu \mathrm{m}$ thick Zn coating and $100 \mu \mathrm{m}$ thick Monel coating, sample thickness $2.28 \mathrm{~mm}$.

For simulations were used He ions and a $15 \mathrm{MeV}$ beam.

\section{Proton irradiation test}

Proton irradiation tests were performed to verify the simulation results. The irradiation tests were conducted at Horia Hulubei National Institute for R\&D in Physics and Nuclear Engineering, using a Tandem Van de Graaff 9MV. A beam with the energy of $15.8 \mathrm{MeV}$ was used to irradiate the samples. The experimental setup was composed of a special vacuum screen on which the composite samples were positioned. The screen allows a number of positions for the sample to be irradiated. Considering the goal of the proposed experiment, the available conditions and the economical factor, the vacuum screen was positioned in front of a Faraday cup. With the screen fully retracted, the proton beam would target directly the Faraday cup, while in the other chosen position, the proton beam would target directly the sample. The tests were performed inside the
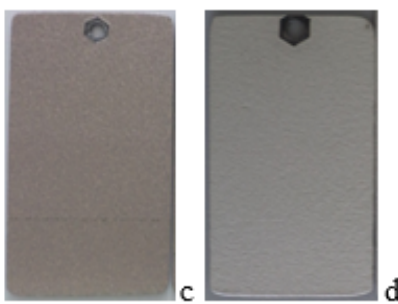

Fig. 3. Composite laminates metallic coated: a) laminates during the coating process; b) laminate coated with $\mathrm{Zn}$;

c) laminate coated with Babbitt;

d) laminate coated with Zn/Monel

vacuum section between an analyser and a switching magnet. The pressure inside the vacuum sections was measured at $3.10^{-6}$ bar and the irradiation time per sample was between $15 \div 30$ s. The Faraday cup measured currents in the range of 3.8 and $4.6 \mu \mathrm{A}$ with the composite samples retracted, while the measured value when the targets were fully inserted was null.

\section{Results and disscusions}

\section{Proton irradiation simulation}

The results of the simulation are presented in figure 4. It ca be observed that in case of using an aluminium sample $2 \mathrm{~mm}$ thick the beam passes through $65 \%$ of the sample $(\sim 1.3 \mathrm{~mm})$. In case of the reference samples made of CFRP it was observed that the ion beam passes through both of the samples, the difference between them was the thickness. Therefore, in case of the $1.9 \mathrm{~mm}$ thick sample the beam passes through entirely and in case of the $2 \mathrm{~mm}$ thick sample the beam passes through partially. All the designs that comprise the Low Z - High Z - Low Z concept stopped the ion beam and the metallic coating ensures a reduction of the penetration depth of the beam. In case of a CFRP sample with Ta foil the beam's penetration depth is $75 \%$ of the sample's thickness $(\sim 1.56 \mathrm{~mm})$, the beam's penetration depth for the CFRP sample with Babbitt coating was $58 \%$ of the sample's thickness $(\sim 1.32 \mathrm{~mm})$, $56 \%$ for the CFRP sample with Zn coating $(\sim 1.28 \mathrm{~mm})$ and $55 \%$ for the CFRP sample with Zn/Monel coating $(\sim 1.25 \mathrm{~mm})$. The sample with the graded metallic coating ensures a higher attenuation of the ion beam, it seems that this material configuration provides an ion beam protection similar with the aluminum sample. The beam's
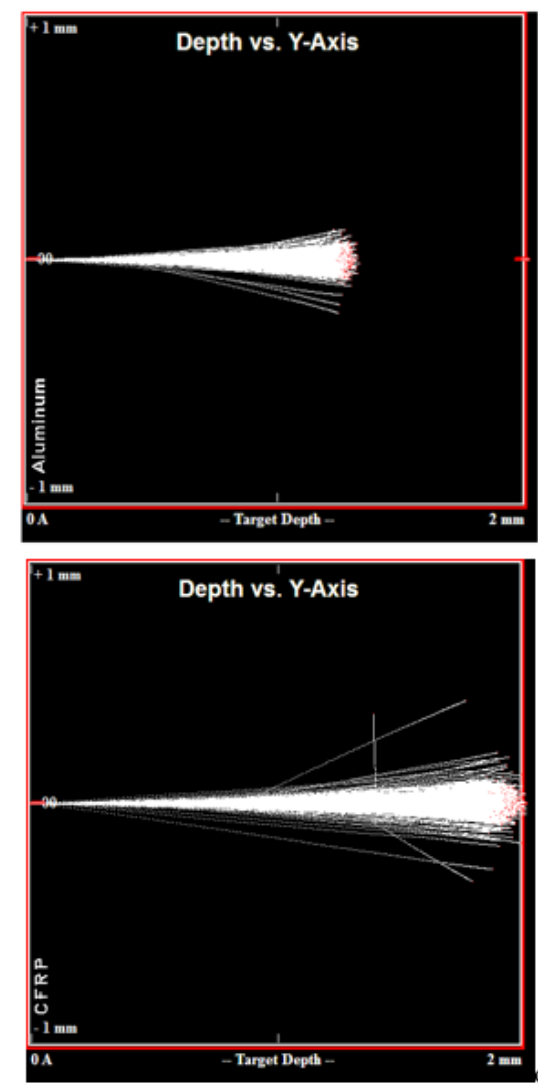

MATERIALE PLASTICE • 55 No. $1 \bullet 2018$
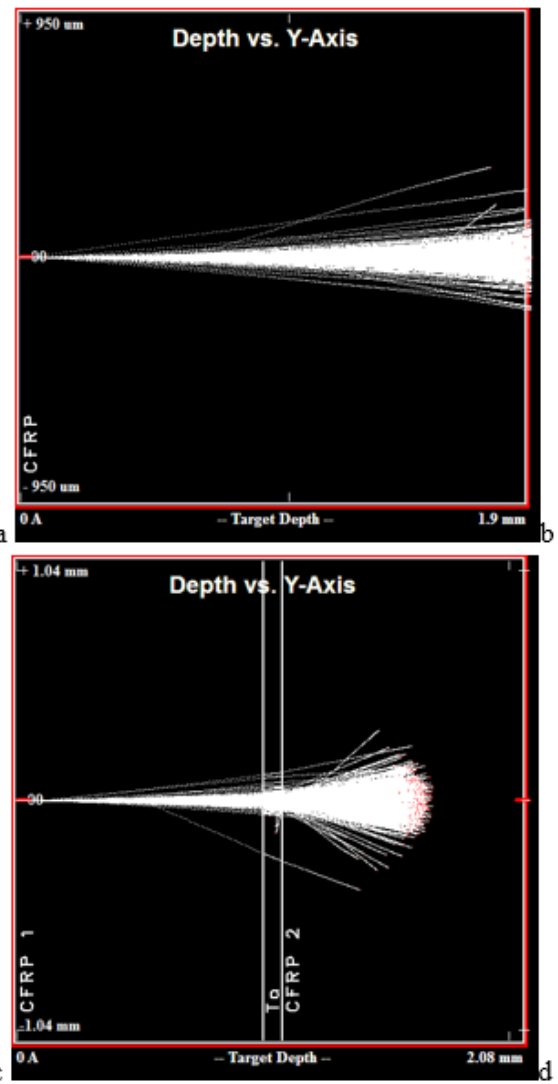

http://www.revmaterialeplastice.ro
Fig. 4. Diagrams resulted from SRIM simulation with a ion beam of $15 \mathrm{MeV}$ 

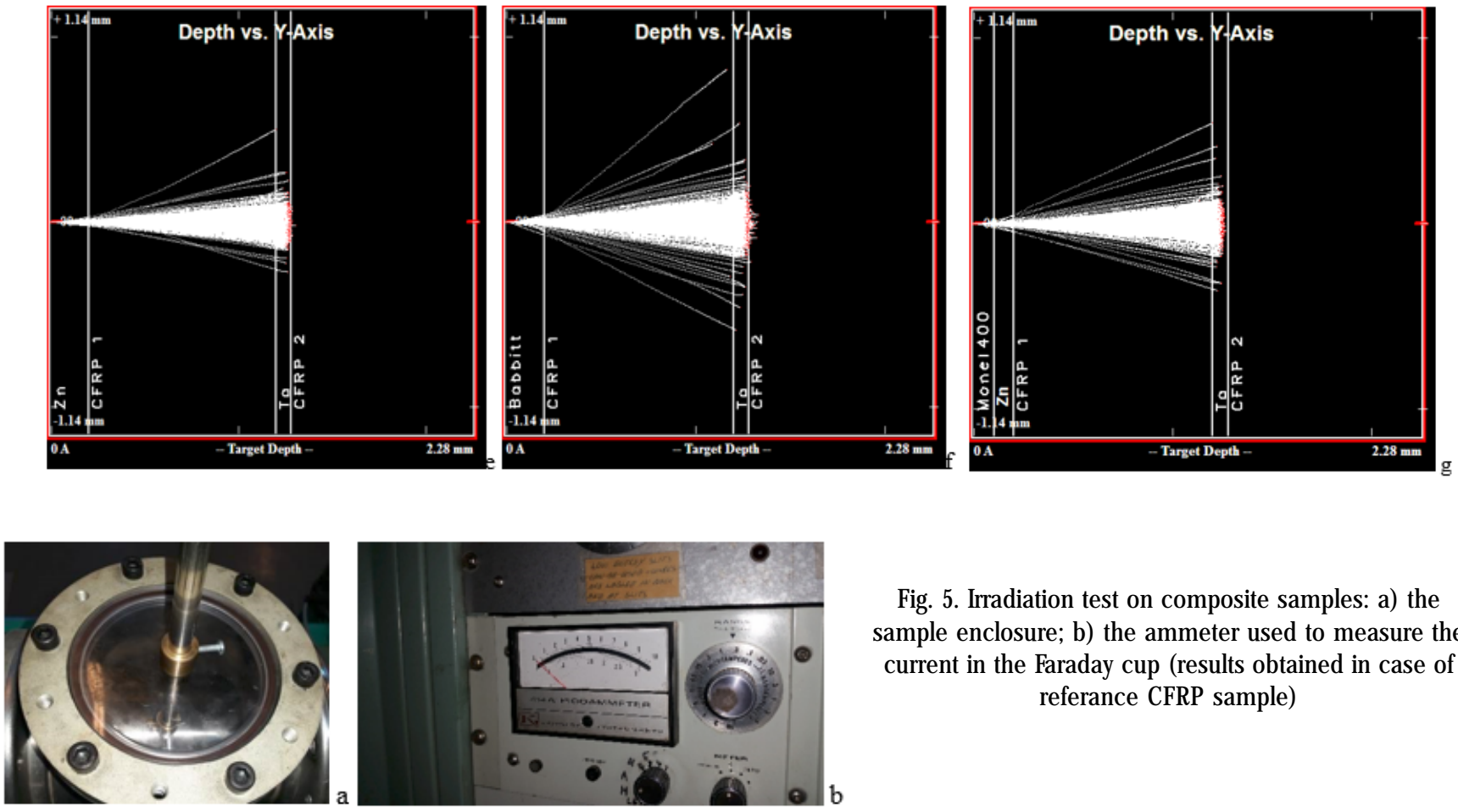

Fig. 5. Irradiation test on composite samples: a) the sample enclosure; $b$ ) the ammeter used to measure the current in the Faraday cup (results obtained in case of referance CFRP sample)

penetration depth in CFRP composite designs is influenced very much by the high $\mathrm{Z}$ material.

\section{Proton irradiation test}

The irradiation test on composite samples confirmed the simulation results, the composite reference sample made only from CFRP was the only configuration penetrated by the ion beam. In this case, the Faraday cup picked-up a current of $3.8 \mu \mathrm{A}$ with and without the target. The current measured by the Faraday cup whithout the target coated with Babbitt was $4.2 \mu \mathrm{A}$, but it wasn't registred current with the target in place. The current measured by the Faraday cup whithout the target coated with Zn/Monel was $4 \mu \mathrm{A}$ and as in the previous case when the target was installed it wasn't registred current. The current measured by the Faraday cup whithout the target coated with $\mathrm{Zn}$ was $4.6 \mu \mathrm{A}$ and when the target was installed it wasn't registred current.

\section{Conclusions}

The materials used to manufacture spacecraft structural components have to meet the requirements generated by the space environment. Radiation shielding is one of the main requirements for the structural components especially if they are used as electronic enclosures. In the present study advanced composite material designs were manufactured. The samples were realised with carbon fiber prepreg fabric preimpregnated with an epoxy cyanate ester blend (cyanat ester resin is commonly used to manufacture space components for spacecraft and satellites). The material designs included the Low Z - High Z - Low Z concept (tantalum foil) and metallic coatings. Ion beam simulations were performed prior the experimental testing and it was observed that all the designs that comprise the Low Z- High Z-Low Z concept stopped the ion beam and the metallic coating ensures a reduction of the penetration depth of the beam $(75 \%$ of the CFRP-Ta sample's thickness, $58 \%$ of CFRP-Ta/Babbitt sample's thickness, $56 \%$ of CFRPTa/Zn sample's thickness, $55 \%$ of CFRP-Ta/Zn/Monel sample's thickness). Experimental was established that the simulation results were accurate, and the CFRP samples with tantalum foil and metallic coating do not allow the proton beam to penetrate them.
Acknowledgement: This work was carried out within Partnerships in priority areas Program-PN II, conducted with the support of MENUEFISCDI, Project no. 293/2014 and Project NUCLEU, Contract no. $11 N / 2016$.

\section{References}

2. *** ISO 15856/2010 - Space systems-Space environment-Simulation guidelines for radiation exposure of non-metallic materials.

2.FRY, R.J.M., Guidance on radiation received in space activities, National Council on Radiation Protection and Measurements: Bethesda, MD, 1989, Quoted in [5]

3. CURTIS, S.B, ATWELL, W., BEEVER, R., HARDY, A., Radiation environments and absorbed dose estimations on manned space missions. Adv. Space Res, 6 , nr. 11, 1986, p. 269, Quoted in [5] 4.KOERSELMAN, J.R., A Multidisciplinary Optimization of Composite Space Enclosures, Master of Science Thesis, Delft University of Technology, 2012

5.NELSON, A.J., Effects of Proton Irradiation on the Mechanical and Physical Properties of Carbon Nanotube Based Composites, Master of Science Thesis, Virginia Polytechnic Institute and State University, 2013

6. BOURDARIE, S., XAPSOS, M., The Near-Earth Space Radiation Environment, Nuclear Science, IEEE Transactions on Nuclear Science, 55, nr. 4, 2008, p. 1810, Quoted in [4]

7. ACKERMANN, M., AJ ELLO, M., ALLAFORT, A., BALADINI, L., BALLET, J, BARBIELLINI, G., BARING, M.G., BASTIERI, D., et.al. - Detection of the characteristic pion-decay signature in supernova remnants, Science, 339, nr. 6121, 2013, p. 807

8. KOELLE, D.E., Specific transport costs to GEO - past, present and future, Acta Astronaut., 53, nr. 4-10, 2003, p. 797

9. CRISP, N. H., SMITH, K.L., HOLLINGSWORTH, P. M., Small Satellite Launch to LEO: A Review of Current and Future Launch Systems, T. Jpn. Soc. Aeronaut.S., 12, 2014, DOI:10.2322/tastj.12.Tf_39

10. AXTEGA, G., MARCOS, J., CARAPELLE, A., ORAVĀ, R.-Radiation shielding of composite space enclosures, 63rd INTERNATIONAL ASTRONAUTICAL CONGRESS, Italy, 2012

11. MANGERET, R., CARRIERE, T., IEEE Transactions on Nuclear Science, 43, nr. 6, 1996, Quoted in [4]

12. BRANDER, T., KRISTOF, G., KATAJ ISTO, H., WALLIN, M. - CFRP electronics housing for a satellite, European Conference on Spacecraft Structures, Materials \& Mechanical Testing, The Netherlands, 2005

Manuscript received: 3.02 .2017 